\title{
A MAXIMUM PRINCIPLE FOR SUBHARMONIC AND PLURISUBHARMONIC FUNCTIONS
}

\author{
CHEN HUAIHUI AND P. M. GAUTHIER
}

\begin{abstract}
We give a simple description of boundary sets which may be ignored in calculating the maximum of subharmonic or plurisubharmonic functions.
\end{abstract}

Let $\bar{R}^{n}=R^{n} \cup\{\infty\}$ denote the one-point compactification of $R^{n}$ and let $\Omega$ be an open subset of $R^{n}$. If $s$ is a function defined on $\Omega$ and $p \in \partial \Omega$, we write

$$
\bar{s}(p)=\varlimsup_{q \rightarrow p} s(q) .
$$

In [4] we find the following characterization of those $\Omega$ for which the supremum of a subharmonic function is always equal to its supremum on the (finite) boundary of $\Omega$.

THEOREM GGH. In order that

$$
\sup _{\Omega} s=\sup _{\partial \Omega \backslash\{\infty\}} \bar{s}
$$

for each subharmonic function s on $\Omega$, it is necessary and sufficient that $\infty$ not be accessible from $\Omega$.

Sahakian [5] independently obtained a more precise result in the case of $R^{2}$ by showing that not only the point at infinity, but indeed, any closed inaccessible portion of the boundary, may be ignored when calculating the supremum.

THEOREM S. Let $\Omega \subset R^{2}$ and let $E$ be a closed subset of $\partial \Omega$. In order that

$$
\sup _{\Omega} s=\sup _{\partial \Omega \backslash E} \bar{s}
$$

for each subharmonic function $s$ on $\Omega$, it is necessary and sufficient that $E$ is not accessible from $\Omega$.

Actually, Theorem GGH was stated in a more general form on manifolds which, as was pointed out in [4], implies Theorem S. Our purpose in this note is to generalize Theorem S sufficiently to have the reverse implication. Moreover, our method will be very simple and different from that employed in [4].

This research was supported by NSERC (Canada), FCAR (Quebec), and NSFC (China).

Received by the editors November 19, 1990 .

AMS subject classification: 30C80, 31B05, 31C10, 31C12.

(C) Canadian Mathematical Society 1992. 
Part of this research was done while the first author was visiting the Centre de Recherches Mathématiques de l'Université de Montréal and later, while the second author was visiting Nanjing Normal University under the sponsorship of the World Bank. We thank all of these institutions.

Let $\Omega$ be a manifold and $\bar{\Omega}$ a compactification of $\Omega$. That is, $\bar{\Omega}$ is a compact Hausdorff space containing (a homeomorphic copy of) $\Omega$ as a dense open subset; the set $\partial \Omega=\bar{\Omega} \backslash \Omega$ is called the ideal boundary of $\Omega$ (relative to $\bar{\Omega}$ ). The compactification $\bar{\Omega}$ is said to be second countable if it has a countable basis. A subset $E$ of the ideal boundary is said to be accessible (from $\Omega$ ) if there is a path in $\Omega$ which is eventually in each neighbourhood of $E$, that is, there is a continuous mapping $\sigma:[0, \infty) \rightarrow \Omega$ such that for each neighbourhood $V$ of $E$, there is a $t^{\prime} \in[0, \infty)$ such that $\sigma(t) \in V$, for each $t>t^{\prime}$. A function $s$, defined on $\Omega$ and taking its values in the extended-reals is said to satisfy the local maximum principle if $s$ is constant in a neighbourhood of each point at which $s$ attains a local maximum. For $p \in \partial \Omega, \bar{s}(p)$ will be defined by formula (1). If $s$ is upper semicontinuous on $\Omega$, then the function defined as $s$ on $\Omega$ and $\bar{s}$ on $\partial \Omega$ is the least upper semicontinuous extension of $s$ to $\bar{\Omega}$.

THEOREM. Let $\bar{\Omega}$ be a second countable compactification of a manifold $\Omega$. Suppose $E \subset \partial \Omega$ is not accessible from $\Omega$. Then, for each upper semicontinuous function s on $\Omega$, satisfying the local maximum principle, we have

$$
\sup _{\Omega} s=\sup _{\partial \Omega \backslash E} \bar{s}
$$

and

$$
\sup _{E} \bar{s}=\varlimsup_{\substack{p \rightarrow E \\ p \in \partial \Omega \backslash E}} \bar{s}(p)
$$

COROLlARY. Let $\Omega$ be a Riemannian (respectively complex) manifold and let $\bar{\Omega}$ and $E$ be as in the theorem. Then, (i) and (ii) hold for each function s subharmonic (respectively plurisubharmonic) on $\Omega$.

REMARK 1. Maximum principles on certain unbounded domains also follow from Phragmén-Lindelöf-type theorems. In such theorems the functions under consideration are always assumed to be of restriced growth, however, in our theorem, there is no such restriction. In certain special situations, conclusions similar to the corollary can be easily obtained using arguments employing harmonic measure. Such arguments work only for bounded functions. Again, we do not assume that our functions are bounded. Rather, we infer that they are bounded, given that they are bounded along a certain portion of the boundary. Actually the maximum principle for upper semicontinuous functions satisfying the local maximum principle is equivalent to the maximum principle for bounded such functions. However, such is not the case for subharmonic or plurisubharmonic functions. 
REMARK 2. Our theorem has a converse. Namely, if $E$ is closed and accessible from $\Omega$, then it is trivial to construct an upper semicontinuous function satisfying the local maximum principle for which (i) and, consequently, (ii) fails. Thus, we have, in fact a complete characterization of closed boundary sets which can be disregarded in the maximum principle. If, moreover, $\Omega$ is a Riemannian manifold, then using recent results on extension and approximation [3], one can obtain such a function which is subharmonic and even [1] harmonic.

REMARK 3. The hypothesis of our theorem is more general than in Theorem GGH, in which $\partial \Omega$, except $\infty$, is embedded in a manifold. Therefore, our theorem implies Theorem GGH, but the reverse implication is not valid and the method employed in [4] does not work for our case.

Proof of Theorem. Since (i) is an immediate consequence of (ii), we shall prove (ii) only. Set

$$
M=\varlimsup_{\substack{p \rightarrow E \\ p \in \partial \Omega \backslash E}} \bar{s}(p)
$$

We may assume that $M$ is finite. Fix $\varepsilon>0$. We shall show that

$$
\sup _{E} \bar{s} \leq M+\varepsilon .
$$

We shall assume, at first, that $E$ is closed. In order to study the behaviour of $s$ near $E$, it is natural to construct a convenient neighbourhood system of $E$.

LEMMA 1. The compact set E has a countable neighbourhood system $V_{1} \supset V_{2} \supset \cdots$ such that $V_{n} \supset \bar{V}_{n+1}$, for $n=1,2, \ldots$, and there is a locally finite family $\beta$ of open sets in $\Omega$ such that for each $n=1,2, \ldots, \Omega \cap V_{n}$ is a union of sets in $\beta$.

Let $A$ be a countable basis for the topology of $\bar{\Omega}$. Let $A^{\prime}$ be the family of sets $\bigcup_{i \in I} a_{i}$ which cover $E$, where $\left\{a_{i}: i \in I\right\}$ are finite families in $A$. Then $A^{\prime}$ is a countable neighbourhood system of $E$, from which we can easily construct a nested countable neighbourhood system $G_{1} \supset G_{2} \supset \cdots$. Since $\bar{\Omega}$ is compact, we may further assume that $G_{n} \supset \bar{G}_{n+1}$, for $n=1,2, \ldots$.

Since $\bar{\Omega}$ is second countable, so is $\Omega$ and since, moreover, $\Omega$ is locally Euclidean, it is $\sigma$-compact and hence paracompact (see $[2,425 \mathrm{X}]$ ). Thus, for $n=2,3, \ldots, \bar{G}_{n} \backslash \partial \Omega$ can be covered by a locally finite family $\beta_{n}$ of parametric balls $B$ such that $B \subset G_{n-1}$. By a parametric ball, we mean an open subset of $\Omega$ whose closure (in $\Omega$ ) is homemorphic to a closed ball. Let $\beta=\bigcup_{n} \beta_{n}$ and for each $n=1,2, \ldots$, set

$$
V_{n}=G_{n+1} \cup \bigcup\left\{B: B \in \beta_{n+1}\right\} .
$$

Then,

$$
\Omega \cap V_{n}=\bigcup\left\{B: B \in \beta_{n+1}\right\}
$$

and the sets $V_{1}, V_{2}, \ldots$ have the properties affirmed in Lemma 1 . 
LEMMA 2. If $V$ is an arbitrary neighbourhood of $E$, then there is a smaller neighbourhood $W$ of $E, W \subset V$, such that

$$
\sup _{\Omega \cap \partial W} s \leq M+\varepsilon .
$$

Let $\left\{V_{n}\right\}$ be a neighbourhood system of $E$ as in Lemma 1 . We may assume that $V \supset V_{1}$ and that

$$
\sup _{\partial \Omega \cap \bar{V}_{1} \backslash E} \bar{s} \leq M+\varepsilon / 2
$$

Set $H_{1}=\Omega \cap V_{1}$ and denote by $X_{1, i}, i=1,2, \ldots$, those components of $H_{1}$ such that

$$
\sup _{\Omega \cap \partial X_{1, i}} s \leq M+\varepsilon,
$$

and by $Y_{1, j}, j=1,2, \ldots$, the other components. Thus, there exists $p_{j} \in \Omega \cap \partial Y_{1, j}$ with $s\left(p_{j}\right)>M+\varepsilon$. We claim that there are only finitely many $Y_{1, j}$. Indeed, if not, then there exists a sequence $p_{j}$ which we may assume converges to some point $p \in \partial V_{1}$. Thus $p \notin E$ since $E \subset V_{j}$. Moreover, $p \notin \partial \Omega \backslash E$ by (3). Hence $p \in \Omega$. From Lemma 1, it follows that there is a neighbourhood of $p$ which meets only finitely many components of $H_{1}$. This contradicts the statement that the sequence $\left\{p_{j}\right\}$ converges to $p$. Hence, there are only finitely many $Y_{1, j}$ as claimed. Set

$$
H_{2}=H_{1} \cap V_{2} \backslash \bigcup_{i} X_{1, i}=\Omega \cap V_{2} \backslash \bigcup_{i} \bar{X}_{1, i} .
$$

We redo the same procedure for $H_{2}$. That is, we divide the components into two classes $X_{2, i}, 1,2, \ldots$ and $Y_{2, j}, j=1,2, \ldots$ as above. We set

$$
H_{3}=H_{2} \cap V_{3} \backslash \bigcup_{i} X_{2, i}=\Omega \cap V_{3} \backslash \bigcup_{i} \bar{X}_{2, i} .
$$

We do this for each $n=2,3, \ldots$. Each component of $H_{n}$ is also a component of $\Omega \cap V_{n}$ and so the components of $H_{n}$ are a locally finite family. Thus, the same argument as at the first stage shows that there are at most finitely many $Y_{n, j}$ for each $n$.

Each $Y_{n+1, j}$ is contained in some component of $H_{n}$. But the $X_{n, i}$ have been removed. Thus, each $Y_{n+1, j}$ is contained in some $Y_{n, k}$. We say that $Y_{n+1, j}$ is a descendant of $Y_{n, k}$ in this case. Let us agree that a descendant of a descendant is also a descendant and so on to any number of generations.

If there are $Y_{n, j}$ for each stage $n$, then since there are only finitely many at the first stage, we may pick some $Y_{1, j}$, denote it by $Y_{1}$ such that $Y_{1}$ has infinitely many descendants. Now $Y_{1}$ has only finitely many descendants $Y_{2, j}$ at the second stage, and so we may choose one, denote it by $Y_{2}$, such that $Y_{2}$ has infinitely many descendants. Continuing in this manner, since at each stage there are at most finitely many $Y_{n, j}$, we construct a sequence $Y_{1} \supset Y_{2} \supset \cdots$, where each $Y_{n}$ is some $Y_{n j}$. 
Now for each $n$, choose a point $q_{n} \in Y_{n}$ and construct a path which starts from $q_{1}$ and goes to $q_{2}$ within $Y_{1}$, then goes to $q_{3}$ within $Y_{2}$ etc. This path is eventually in each $Y_{n}$ and, ipso facto, in each $V_{n}$. This contradicts the inaccessibility of $E$. Thus, at some stage $m$, there are no $Y_{m, j}$. Hence

$$
H_{m}=H_{m-1} \cap V_{m} \backslash \bigcup_{i} \bar{X}_{m-1, i}=\bigcup_{i} X_{m, i} .
$$

Set

$$
W=V_{m} \cup \bigcup_{n<m} \bigcup_{i} X_{n, i} .
$$

Then, $W$ is a neighbourhood of $E$ contained in $V$,

$$
\Omega \cap W=\bigcup_{n \leq m} \bigcup_{i} X_{n, i},
$$

and since $X_{n, i}$ form a locally finite family,

$$
\Omega \cap \partial W=\bigcup_{n \leq m} \bigcup_{i}\left[\Omega \cap \partial X_{n, i}\right]
$$

From the definition of the $X_{n, i}$, Lemma 2 follows.

By combining Lemma 1, Lemma 2 , and the definition of $M$, we may construct a neighbourhood system of $E: W_{1} \supset W_{2} \supset \cdots$ such that

$$
\sup _{\left(\bar{W}_{1} \cap \partial \Omega\right) \backslash E} s \leq M+\varepsilon,
$$

and for $n=1,2, \ldots$

$$
W_{n} \supset \bar{W}_{n+1^{\prime}}
$$

and

$$
\sup _{\Omega \cap \partial W_{n}} s \leq M+\varepsilon
$$

Since $s$ satisfies the local maximum principle, it follows from (4) and (5) that

$$
\sup _{\Omega \cap\left[W_{n} \backslash \bar{W}_{n+1}\right]} s \leq M+\varepsilon,
$$

for $n=1,2, \ldots$. This proves (2).

Suppose now that $E$ is not closed. Let $W$ be an open neighbourhood of $E$ such that

$$
\sup _{(W \cap \partial \Omega) \backslash E} \bar{s}<M+\varepsilon,
$$

and set

$$
E(\varepsilon)=\{p \in W \cap \partial \Omega: \bar{s}(p) \geq M+\varepsilon\} .
$$

Then $E(\varepsilon)$ is a closed subset of $E$. By the result proved above for a closed set,

$$
\sup _{E(\varepsilon)} \bar{s} \leq M+\varepsilon .
$$

By (6), (7), and (8), (2) is also proved.

Let $\varepsilon \rightarrow 0$ in (2); we obtain (ii). This completes the proof of the theorem. 


\section{REFERENCES}

1. T. Bagby and P. Blanchet, Uniform harmonic approximation on Riemannian manifolds, J. d'Analyse Math., (to appear).

2. Encyclopedic Dictionary of Mathematics, 2nd ed., MIT Press, Cambridge, 1987.

3. P. M. Gauthier, Subharmonic and plurisubharmonic extensions and approximations, manuscript.

4. P. M. Gauthier, R. Grothmann, and W. Hengartner, Asymptotic maximum principles for subharmonic and plurisubharmonic functions, Can. J. Math. 40(1988), 477-486.

5. R. Sh. Sahakian, On a generalization of the maximum principle (Russian), Izv. Akad. Nauk Arm. SSR Mat. 22(1987), 94-101; translation in Soviet J. Contemporary Math. Anal. 22(1987), 94-102.

Mathematics Department

Nanjing Normal University

Nanjing, China

Département de mathématiques et de statistique

Université de Montréal, C.P. 6128

Montréal, Quebec

H3C $3 J 7$ 